\title{
232 INCB090244, A POTENT SMALL MOLECULE THAT INHIBITS THE PD-L1/PD-1 AXIS AND FUNCTIONS SIMILARLY TO PD-L1 ANTIBODIES
}

\footnotetext{
${ }^{1} J o n a t h a n$ Rios-Doria*, 'Alla Volgina, ${ }^{2}$ Prafulla Gokhale, ${ }^{1}$ Hao Liu, ${ }^{1}$ Christina Stevens, ${ }^{1}$ Nina Zolotarjova, ${ }^{1}$ Darlise DiMatteo, ${ }^{1}$ Kanishk Kapilashrami, ${ }^{1}$ Elham Behshad, ${ }^{1}$ Pramod Thekkat, 'Gengjie Yang, 'Leslie Hall, 'Chrysi Kanellopoulou, 'Mark Rupar, ${ }^{1}$ Christopher Maddage, ${ }^{1}$ April Horsey, ${ }^{1}$ Krista Burke, ${ }^{1}$ Yan-ou Yang, ${ }^{1}$ Maryanne Covington, ${ }^{1}$ Steve Wang, ${ }^{1}$ Phillip Liu, ${ }^{1}$ Richard Wynn, ${ }^{2}$ David Reardon, ${ }^{1}$ Holly Koblish. 'Incyte Corporation, Wilmington, USA; ${ }^{2}$ Harvard, Boston, MA, USA
}

Background Blocking the PD-L1 immune checkpoint axis with therapeutic antibodies against either the ligand or PD-1 has proven to be an effective treatment modality for multiple cancer histologies. Small molecules targeting the PD-L1/PD-1 axis represent an alternate modality of blocking this pathway. INCB090244 is a small molecule that blocks the PD-L1/PD-1 interaction and restores $\mathrm{T}$ cell function similar to the clinical stage PD-L1 inhibitor INCB086550.

Methods MDA-MB-231 or CHO cells overexpressing PD-L1 were used to investigate effects of INCB090244 on PD-L1 dimerization, and intracellular trafficking. In vivo, CD34+ humanized mice harboring MDA-MB-231 tumors or C57Bl/6 mice bearing GL261 subcutaneous or orthotopic tumors were used to investigate the efficacy, biodistribution, and pharmacodynamic effects of INCB090244. Human specific gene expression changes in tumors from MDA-MB-231 bearing humanized mice were analyzed by RNA sequencing.

Results In vitro, INCB090244 potently disrupted the PD-L1: PD-1 interaction, induced PD-L1 dimerization, and inhibited PD-1-mediated negative signaling, resulting in enhanced IFN gamma and IL-2 production in primary human immune cells. Following dimerization, INCB090244 induced internalization of PD-L1 resulting in co-localization with the Golgi apparatus and partial localization in the nucleus. After cell treatment and washing, full restoration of PD-L1 at the cell surface was observed after 5 days of culture in vitro. In vivo, INCB090244 reduced tumor growth in CD34+ humanized mice bearing MDA-MB-231 tumors, to similar levels as atezolizumab. Antitumor activity was completely abrogated in immunodeficient mice, confirming the pharmacologic dependency on a competent immune system. RNA sequencing analysis on tumors from these mice demonstrated similar $\mathrm{T}$ cell activation gene signatures as clinical checkpoint blockade antibodies. Biodistribution studies in mice bearing both subcutaneous and orthotopically implanted GL261 glioma tumors demonstrated higher accumulation of INCB090244 in tumor tissue compared to PD-L1 antibodies.

Conclusions INCB090244 effectively disrupted the PD-L1/PD1 interaction, induced dimerization and internalization of PDL1, restored immunity in in vitro and in vivo tumor models, and is a suitable surrogate for the clinical candidate INCB086550. RNA sequencing demonstrated T cell activation signatures similar to those observed in patients receiving checkpoint blockade antibodies. Biodistribution studies demonstrated higher subcutaneous and brain tumor penetration by INCB090244 compared to PD-L1 antibodies, suggesting a potential advantage of small molecule PD-L1 inhibitors in accessing intratumoral regions. These data further support the clinical evaluation of small molecule PD-L1 inhibitors as an alternative approach to immune therapy.

http://dx.doi.org/10.1136/jitc-2021-SITC2021.232 